\title{
Not Completely Innocent: How Argon Binding Perturbs Cationic Copper Clusters
}

\author{
Zahra Jamshidi*a,b, Olga V. Lushchikovac, Joost M. Bakker*c, and Lucas Visscher*b \\ ${ }^{a}$ Chemistry Department, Sharif University of Technology, Tehran 11155-9516, Iran \\ ${ }^{b}$ Theoretical Chemistry, Vrije Universiteit Amsterdam, The Netherlands \\ c Radboud University, Institute for Molecules and Materials, FELIX Laboratory, Nijmegen, The Netherlands

\section{SUPPORTING INFORMATION}

\section{(9 pages)}

Table S1. The values of $\Delta \mathrm{H}_{\mathrm{n}, \mathrm{m}}(\mathrm{m}=1-\mathrm{n})$ (in $\mathrm{eV}$ ) for detaching an Ar atom at $180 \mathrm{~K}$

Table S2. The binding energies (in eV) at different level of theory.

Table S3. Energy decomposition analysis (in eV) for $\mathrm{Cu}_{1,2}{ }^{+} \mathrm{Ar}_{\mathrm{m}}$ interactions at PBE-D3/TZ2P

level of theory.

Table S4. Optimized coordinates of $\mathrm{Cu}_{n}{ }^{+} \mathrm{Ar}_{\mathrm{m}}(\mathrm{n}=1-7)$ at PBE-D3/TZ2P level of theory. .S4

Figure S1. Natural population analysis of bare $\mathrm{Cu}_{\mathrm{n}}{ }^{+}(\mathrm{n}=3-7)$ clusters............................... 9

Figure S2. Natural population analysis of $\mathrm{Cu}_{\mathrm{n}}{ }^{+} \mathrm{Ar}(\mathrm{n}=3-7)$ complexes. S9 
Table S1. The values of $\Delta \mathrm{H}_{\mathrm{n}, \mathrm{m}}(\mathrm{m}=1-\mathrm{n})$ (in eV) for detaching an Ar atom at $180 \mathrm{~K}$.

\begin{tabular}{|c|c|c|c|c|c|c|c|c|c|c|c|c|c|}
\hline Complex & $\Delta H_{7, m}$ & Complex & $\Delta H_{6, m}$ & Complex & $\Delta H_{5, m}$ & Complex & $\Delta H_{4, m}$ & Complex & $\Delta H_{3, m}$ & Complex & $\underset{\mathrm{a}}{\Delta H_{2, k}}$ & Complex & $\begin{array}{l}\Delta l \\
\mathrm{a}\end{array}$ \\
\hline$C u_{7}^{+A r_{7}}$ & -0.112 & & & & & & & & & & & & \\
\hline$C u_{7}^{+A r_{6}}$ & -0.124 & $C u_{6}^{+A r_{6}}$ & -0.125 & & & & & & & & & & \\
\hline$C u_{7}^{+A r_{5}}$ & -0.129 & $C u_{6}^{+A r_{5}}$ & -0.126 & $C u_{5}^{+A r_{5}}$ & -0.085 & & & & & & & $C u^{+A r_{5}}$ & \\
\hline$C u_{7}^{+A r_{4}}$ & -0.139 & $C u_{6}^{+A r_{4}}$ & -0.150 & $C u_{5}^{+A r_{4}}$ & -0.098 & $C u_{4}^{+A r_{4}}$ & -0.188 & & & $C u_{2}^{+A r_{4}}$ & -0.144 & $C u^{+A r_{4}}$ & 0.2 \\
\hline$C u_{7}^{+A r_{3}}$ & -0.137 & $C u_{6}^{+A r_{3}}$ & -0.146 & $C u_{5}^{+A r_{3}}$ & -0.330 & $C u_{4}^{+A r_{3}}$ & -0.201 & $C u_{3}^{+A r_{3}}$ & -0.315 & $C u_{2}^{+A r_{3}}$ & -0.166 & $C u^{+A r_{3}}$ & 0.2 \\
\hline$C u_{7}^{+A r_{2}}$ & -0.265 & $C u_{6}^{+A r_{2}}$ & -0.269 & $C u_{5}^{+A r_{2}}$ & -0.352 & $C u_{4}^{+A r_{2}}$ & -0.284 & $C u_{3}^{+A r_{2}}$ & -0.347 & $C u_{2}^{+A r_{2}}$ & -0.355 & $C u^{+A r_{2}}$ & \\
\hline $\mathrm{Cu}_{7}^{+A r}$ & -0.282 & $C u_{6}^{+A r}$ & -0.305 & $C u_{5}^{+A r}$ & -0.360 & $\mathrm{Cu}_{4}^{+A r}$ & -0.306 & $C u_{3}^{+A r}$ & -0.378 & $C u_{2}^{+A r}$ & -0.401 & $C u^{+A r}$ & -0.7 \\
\hline
\end{tabular}

${ }^{\text {a }} k=1-4$ and $j=1-5$

Table S2. The binding energies (in eV) at different level of theory.

\begin{tabular}{cllll}
\hline$\Delta E_{b^{\mathrm{a}}}$ & $\mathrm{MP2}$ & $\mathrm{CCSD}$ & $\mathrm{CCSD}(\mathrm{T})$ & $\mathrm{PBE}-\mathrm{D} 3$ \\
\hline$C u^{+A r}$ & $-0.57(-0.56)$ & $-0.50(-0.46)$ & $-0.56(-0.53)$ & -0.81 \\
$C u^{+A r_{2}}$ & $-0.68(-0.68)$ & $-0.58(-0.53)$ & $-0.66(-0.62)$ & -0.84 \\
$C u^{+A r_{3}}$ & $-0.21(-0.20)$ & $-0.24(-0.22)$ & $-0.24(-0.24)$ & -0.28 \\
$C u^{+A r_{4}}$ & $-0.20(-0.21)$ & $-0.22(-0.20)$ & $-0.22(-0.22)$ & -0.27 \\
$C u^{+A r_{5}}$ & $-0.05(-0.08)$ & $-0.06(-0.08)$ & $-0.06(-0.09)$ & -0.13 \\
$C u_{2}^{+A r}$ & -0.43 & -0.39 & -0.41 & -0.45 \\
$C u_{2}^{+A r_{2}}$ & -0.37 & -0.34 & -0.34 & -0.37 \\
$C u_{2}^{+A r_{3}}$ & -0.13 & -0.09 & -0.07 & -0.22 \\
$C u_{3}^{+A r}$ & -0.41 & -0.34 & -0.39 & -0.43 \\
$C u_{3}^{+A r_{2}}$ & -0.39 & -0.32 & -0.36 & -0.40 \\
$C u_{3}^{+}+r_{3}$ & -0.37 & -0.29 & -0.34 & -0.36 \\
\hline
\end{tabular}

${ }^{\mathrm{a}} \Delta E_{b}=E_{C u_{n}^{+A r_{-}}},{ }^{\mathrm{b}}$ Values in parentheses were computed with the uncontracted triple zeta basis set of Dyall $\underline{\underline{21}}$. 
Table S3. Energy decomposition analysis (in eV) for $\mathrm{Cu}_{1,2}{ }^{+} \mathrm{Ar}_{\mathrm{m}}$ interactions at PBE-D3/TZ2P level of theory.

\begin{tabular}{|c|c|c|c|c|}
\hline Complex & $\Delta E_{\text {Pauli }}$ & $\Delta V_{\text {elstat }}+\Delta E_{o i}$ & $\Delta E_{d i s p}$ & $\Delta E_{\int^{\mathrm{a}}}$ \\
\hline$C u^{+A r_{1}}$ & 1.46 & -2.25 & -0.02 & -0.81 \\
\hline$C u^{+A r_{2}}$ & 1.25 & -2.06 & -0.03 & -0.84 \\
\hline$C u^{+A r_{3}}$ & 1.18 & -1.66 & -0.04 & -0.52 \\
\hline$C u^{+A r_{4}}$ & 1.01 & -1.35 & -0.05 & -0.40 \\
\hline$C u^{+A r_{5}}$ & 0.54 & -0.69 & -0.06 & -0.22 \\
\hline$C u_{2}^{+A r_{1}}$ & 1.08 & -1.52 & -0.03 & -0.46 \\
\hline$C u_{2}^{+A r_{2}}$ & 0.99 & -1.35 & -0.03 & -0.39 \\
\hline$C u_{2}^{+A r_{3}}$ & 0.92 & -1.16 & -0.04 & -0.28 \\
\hline$C u_{2}^{+A r_{4}}$ & 0.88 & -1.06 & -0.05 & -0.23 \\
\hline
\end{tabular}

aDue to the deformation of the fragments relative to their optimal structure, interaction energies for the $C u^{+} A r_{m}$ complexes are more negative than the binding energies of Table S2, which correspond to energy differences between optimized structures. 
Table S4. Optimized coordinates of $\mathrm{Cu}_{n}{ }^{+} \mathrm{Ar}_{\mathrm{m}}(\mathrm{n}=1-7)$ at PBE-D3/TZ2P level of theory.

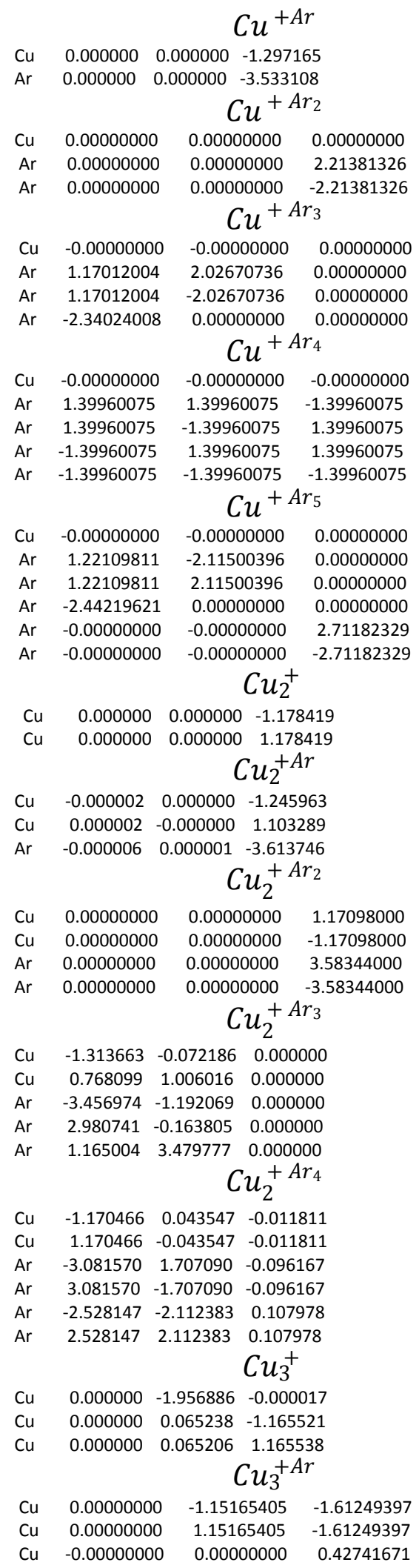




\begin{tabular}{|c|c|c|c|}
\hline $\mathrm{Ar}$ & \multirow[t]{2}{*}{-0.00000000} & \multirow{2}{*}{\multicolumn{2}{|c|}{$\begin{array}{c}0.00000000 \\
C u_{3}^{+} A r_{2}\end{array}$}} \\
\hline & & & \\
\hline $\mathrm{Cu}$ & -0.00000000 & 0.00000000 & 2.08995144 \\
\hline $\mathrm{Cu}$ & -0.00000000 & -1.17724995 & 0.09870796 \\
\hline $\mathrm{Cu}$ & 0.00000000 & 1.17724995 & 0.09870796 \\
\hline $\mathrm{Ar}$ & 0.00000000 & 3.20323496 & -1.14368369 \\
\hline $\mathrm{Ar}$ & -0.00000000 & \multicolumn{2}{|c|}{$C u_{3}^{+}{ }^{+A r_{3}}$} \\
\hline $\mathrm{Cu}$ & -1.34172107 & 0.00000000 & 0.00000000 \\
\hline $\mathrm{Cu}$ & 0.67086053 & -1.16196453 & 0.00000000 \\
\hline $\mathrm{Cu}$ & 0.67086053 & 1.16196453 & 0.00000000 \\
\hline $\mathrm{Ar}$ & 1.86168570 & 3.22453421 & 0.00000000 \\
\hline $\mathrm{Ar}$ & 1.86168570 & -3.22453421 & 0.00000000 \\
\hline $\mathrm{Ar}$ & -3.72337139 & \multicolumn{2}{|l|}{$\begin{array}{r}0.00000000 \\
C u_{4}^{+}\end{array}$} \\
\hline $\mathrm{Cu}$ & -0.000000 & \multicolumn{2}{|c|}{$-2.066116-0.000000$} \\
\hline $\mathrm{Cu}$ & 0.000000 & \multicolumn{2}{|c|}{$0.000001-1.171799$} \\
\hline $\mathrm{Cu}$ & 0.000000 & \multicolumn{2}{|c|}{$0.000001 \quad 1.171799$} \\
\hline $\mathrm{Cu}$ & 0.000000 & \multicolumn{2}{|c|}{$\begin{array}{c}2.066115-0.000000 \\
C u_{4}^{+A r}\end{array}$} \\
\hline $\mathrm{Cu}$ & -0.001660 & \multicolumn{2}{|c|}{$-2.055093 \quad 0.092422$} \\
\hline $\mathrm{Cu}$ & -0.003312 & $0.003231-1.04$ & 787 \\
\hline $\mathrm{Cu}$ & 0.000109 & $0.006300 \quad 1.301$ & 548 \\
\hline $\mathrm{Cu}$ & -0.001648 & 2.0645310 .087 & 050 \\
\hline $\operatorname{Ar}$ & 0.003628 & $\begin{array}{c}0.009385 \\
C u_{4}^{+}\end{array}$ & \\
\hline $\mathrm{Cu}$ & -0.001840 & $-2.048721 \quad 0.10$ & 5942 \\
\hline $\mathrm{Cu}$ & -0.003762 & $0.003194-1.07$ & 5601 \\
\hline $\mathrm{Cu}$ & 0.000094 & $0.006360 \quad 1.28$ & 872 \\
\hline $\mathrm{Cu}$ & -0.001828 & $2.058111 \quad 0.10$ & 1331 \\
\hline $\mathrm{Ar}$ & 0.004103 & $0.009281 \quad 3.740$ & 529 \\
\hline $\mathrm{Ar}$ & -0.007771 & $\begin{array}{r}-0.002720-3.53 \\
C u_{4}^{+}\end{array}$ & \\
\hline $\mathrm{Cu}$ & -0.089791 & $-1.715107-0.03$ & 8206 \\
\hline $\mathrm{Cu}$ & -1.192321 & $0.385346-0.01$ & 1328 \\
\hline $\mathrm{Cu}$ & 1.128171 & $0.304282 \quad 0.22$ & 116 \\
\hline $\mathrm{Cu}$ & 0.023371 & $2.404138 \quad 0.27$ & 226 \\
\hline $\mathrm{Ar}$ & 3.569540 & $0.265731 \quad 0.45$ & 647 \\
\hline $\mathrm{Ar}$ & -3.628211 & $0.520132-0.26$ & 651 \\
\hline $\mathrm{Ar}$ & -0.159281 & $\begin{array}{r}-4.191323-0.20 \\
C u_{4}^{+}\end{array}$ & \\
\hline $\mathrm{Cu}$ & -0.031663 & $-1.716811 \quad 0.11$ & 1315 \\
\hline $\mathrm{Cu}$ & -0.033550 & $0.360998-1.04$ & 9206 \\
\hline $\mathrm{Cu}$ & -0.029768 & $0.364378 \quad 1.26$ & 5741 \\
\hline $\mathrm{Cu}$ & -0.031655 & $2.442206 \quad 0.10$ & 220 \\
\hline $\mathrm{Ar}$ & -0.025746 & $0.368218 \quad 3.72$ & 041 \\
\hline $\mathrm{Ar}$ & -0.037572 & $0.357634-3.51$ & 1506 \\
\hline $\mathrm{Ar}$ & -0.031649 & $4.965969 \quad 0.10$ & 1710 \\
\hline $\mathrm{Ar}$ & -0.031669 & $\begin{array}{r}-4.240579 u_{5}^{+} \\
C .11\end{array}$ & 4826 \\
\hline $\mathrm{Cu}$ & -1.182828 & $\begin{array}{lll}8 & -0.542499 & 0.8\end{array}$ & 59648 \\
\hline $\mathrm{Cu}$ & -0.624942 & $\begin{array}{lll}2 & -0.605123 & -1.5\end{array}$ & 28467 \\
\hline $\mathrm{Cu}$ & 1.068380 & $\begin{array}{lll}-0.711204 & 0.2\end{array}$ & 43558 \\
\hline $\mathrm{Cu}$ & -0.084246 & $\begin{array}{lll}6 & 1.301195 & -0.0\end{array}$ & 75762 \\
\hline $\mathrm{Cu}$ & 0.494507 & $\begin{array}{r}0.634900 \\
C u_{5}^{+}\end{array}$ & 3533 \\
\hline $\mathrm{Cu}$ & -1.134825 & $\begin{array}{lll}5 & -0.559405 & 0.8\end{array}$ & 41325 \\
\hline $\mathrm{Cu}$ & -0.580753 & $\begin{array}{lll}3 & -0.654823 & -1.5\end{array}$ & 34860 \\
\hline $\mathrm{Cu}$ & 1.104361 & $\begin{array}{lll}1 & -0.758623 & 0.2\end{array}$ & 29026 \\
\hline $\mathrm{Cu}$ & -0.017641 & $\begin{array}{lll}1 & 1.281037 & -0.1\end{array}$ & 06444 \\
\hline $\mathrm{Cu}$ & 0.547804 & $\begin{array}{lll}4 & 0.580547 & 2.1\end{array}$ & 3937 \\
\hline $\mathrm{Ar}$ & -0.008483 & $3 \quad 3.539064-0.8$ & 5314 \\
\hline
\end{tabular}




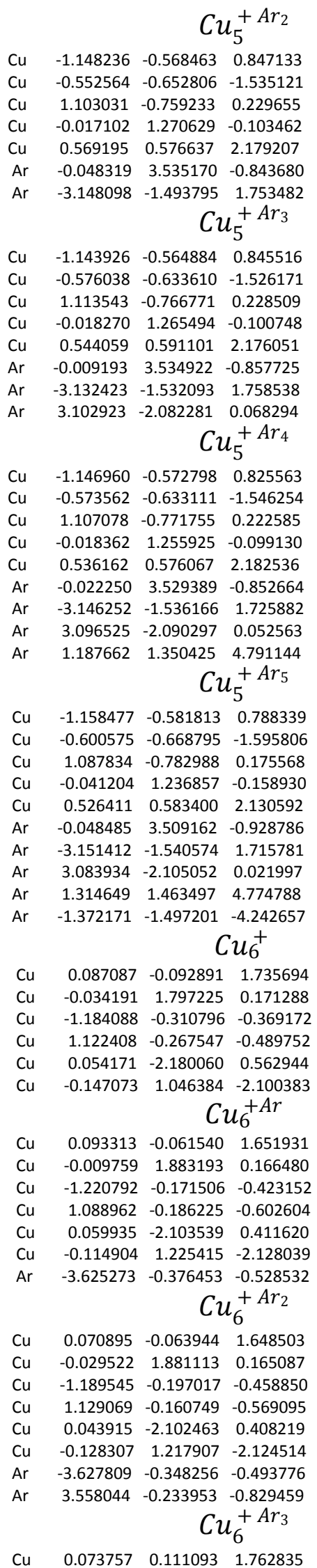




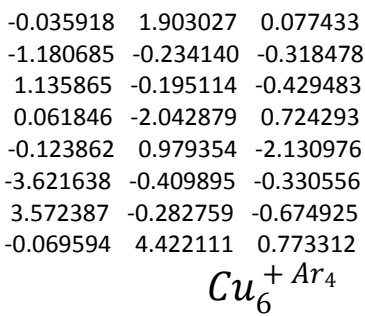




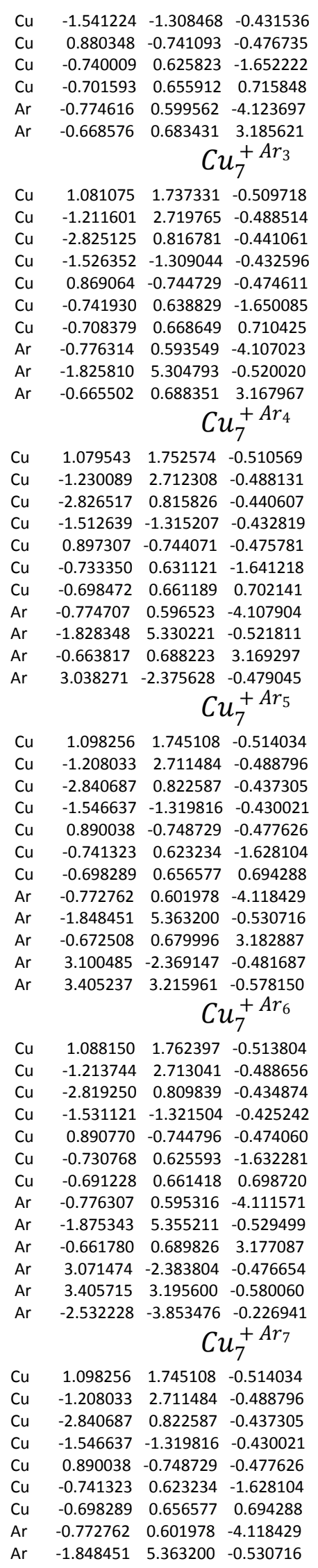


$\begin{array}{llll}\text { Ar } & -0.672508 & 0.679996 & 3.182887\end{array}$

$\begin{array}{llll}\text { Ar } & 3.100485 & -2.369147 & -0.481687\end{array}$

$\begin{array}{llll}\text { Ar } & 3.405237 & 3.215961 & -0.578150\end{array}$

$\begin{array}{llll}\text { Ar } & -2.518292 & -3.868838 & -0.203590\end{array}$

Ar $\quad-5.572843 \quad 0.912356 \quad-0.241623$ 

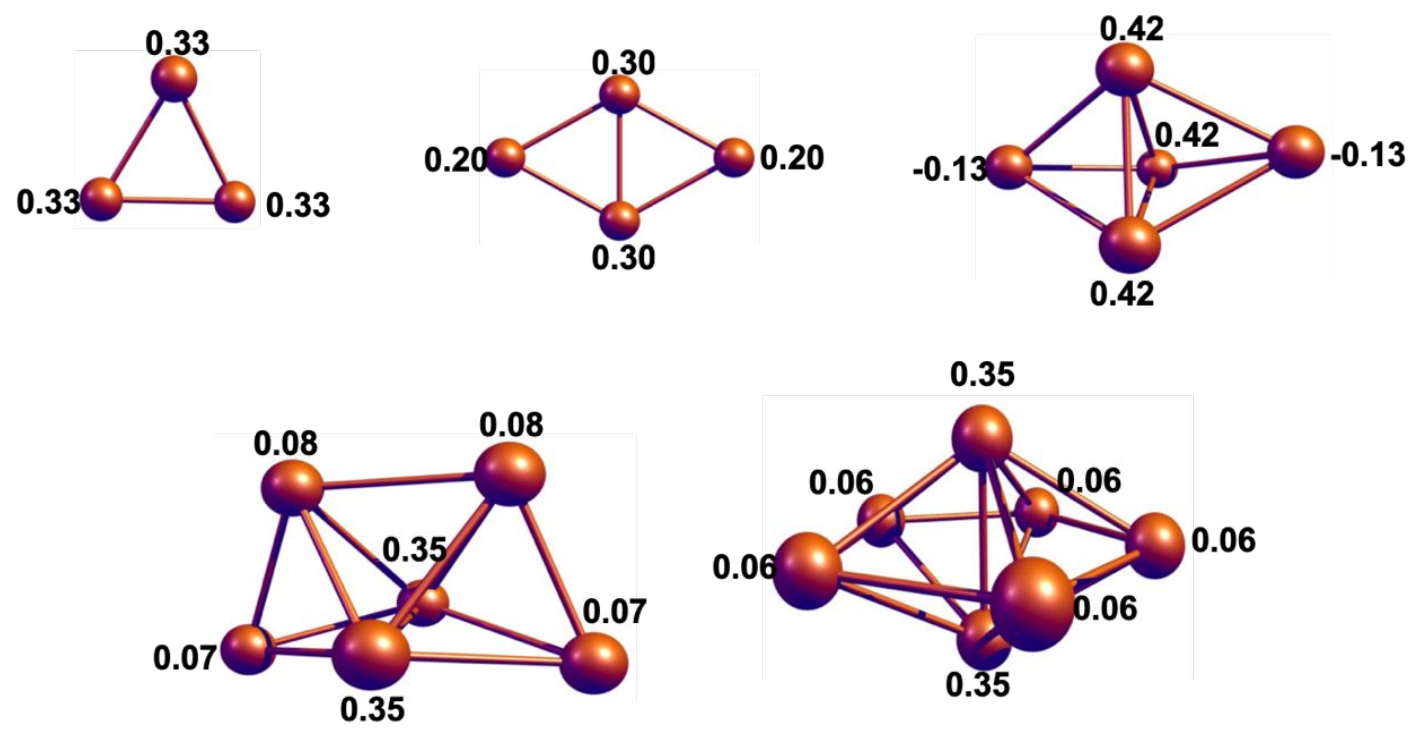

Figure S1. Natural population analysis of bare $\mathrm{Cu}_{n}{ }^{+}(\mathrm{n}=3-7)$ clusters.
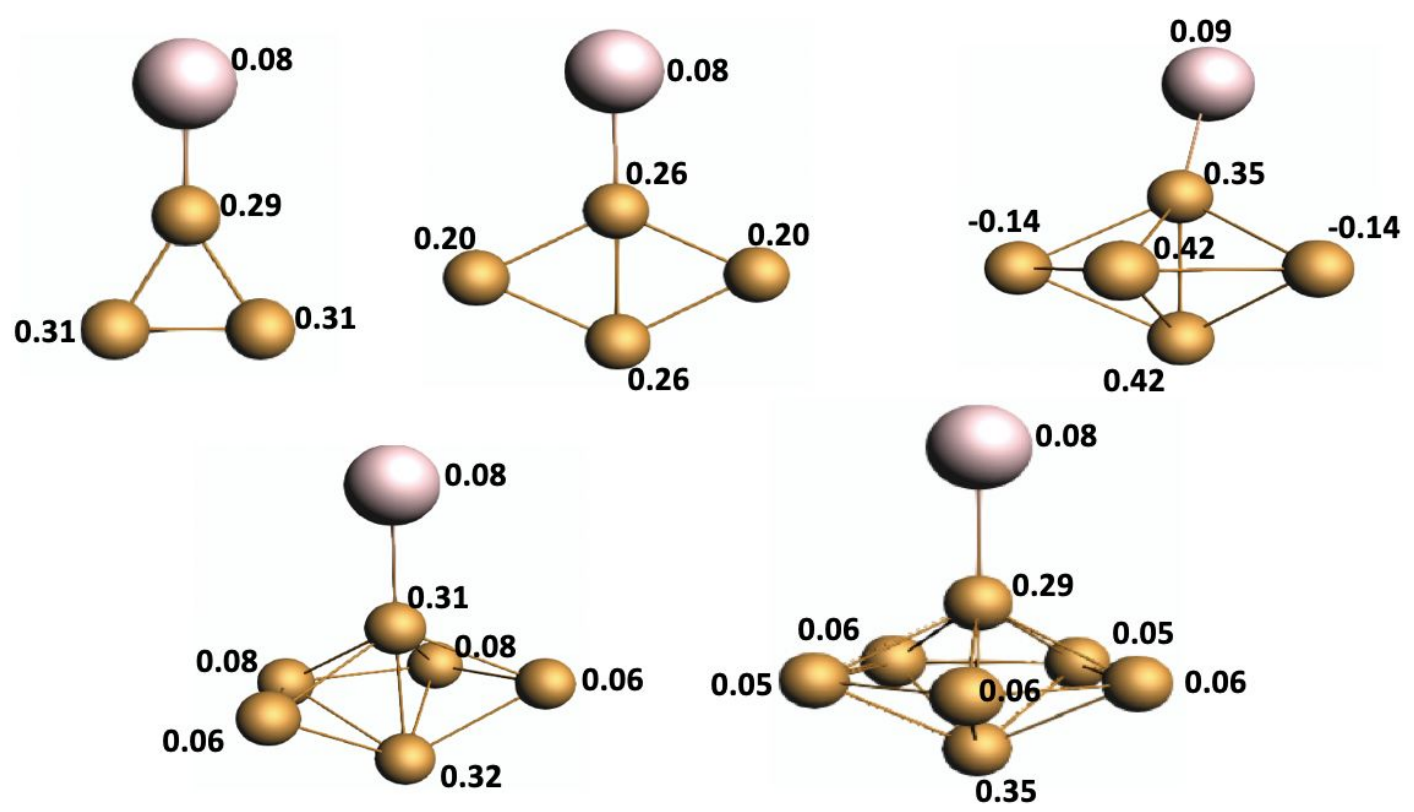

Figure S2. Natural population analysis of $\mathrm{Cu}_{n}{ }^{+} \mathrm{Ar}(\mathrm{n}=3-7)$ complexes. 